\title{
Vimentin is not a reliable prognostic biomarker for cervical cancer
}

\author{
F.C. Santana ${ }^{1}$, J.E.P. Ramos ${ }^{2}$, N.A. Nogueria ${ }^{3}$, L.S.D. Líbera ${ }^{3}$, \\ T. Aparecida ${ }^{4}$, S.H. Rabelo ${ }^{5}$ and V.A. Saddi ${ }^{2,6}$ \\ ${ }^{1}$ Programa de Mestrado em Genética, Pontifícia Universidade Católica de \\ Goiás, Goiânia, GO, Brasil \\ ${ }^{2}$ Programa de Mestrado em Ciências Ambientais e Saúde, Pontifícia \\ Universidade Católica de Goiás, Goiânia, GO, Brasil \\ ${ }^{3}$ Programa de Pós-graduação em Ciências da Saúde, Faculdade de Medicina, \\ Universidade Federal de Goiás, Goiânia, GO, Brasil \\ ${ }^{4}$ Instituto de Epimemiologia e Saúde Tropical, Universidade Federal de \\ Goiás, Goiânia, GO, Brasil \\ ${ }^{5}$ Programa de Pós-graduação em Farmácia, Universidade Federal de Goiás, \\ Goiânia, GO, Brasil \\ ${ }^{6}$ Programa de Mestrado em Ciências Ambientais e Saúde, Faculdade de \\ Medicina, Universidade Federal de Goiás, Goiânia, GO, Brasil \\ Corresponding author: V.A. Saddi \\ E-mail: verasaddi@gmail.com \\ Genet. Mol. Res. 18 (4): gmr18386 \\ Received June 24, 2019 \\ Accepted December 07, 2019 \\ Published December 31, 2019 \\ DOI http://dx.doi.org/10.4238/gmr18386
}

ABSTRACT. Vimentin is a cytoskeletal protein belonging to a
family of intermediate filaments whose expression has been studied
in human cancers due to its association with the mesenchymal
epithelial transition, a cancer reactivation event that results in
complex alterations in the expression of genes involved in the
invasion and metastasis processes. Studies on the prognostic value of
vimentin, using immunohistochemistry are scarce, with conflicting
results. Our evaluation was performed based on 111 cases of cervical
cancer, including different clinical stages and histological types. Our
objective was to evaluate the vimentin expression in cervical cancers,
investigating a possible prognostic role of this biomarker. The
evaluation was performed by immunohistochemistry in cases of
cervical cancer and the marking index was evaluated with regards to 
clinical and pathological aspects, and to survival. Vimentin expression was observed in $100 \%$ of the tumor specimens. Hyperexpression of this biomarker in tumor cells (> 40\%) was observed in $25 \%$ of the cases; however, it was not associated with clinical and pathological, or prognostic aspects of cervical cancer. Five-year survival for this group of patients was 66\%; it was influenced by age, tumor size, presence of lymph node metastases, presence of distant metastases, and clinical stage. Hyperexpression of vimentin was not found to be a prognostic factor for cervical cancer.

Key words: Immunohistochemistry; Survival; Epithelial-mesenchymal transition; Vimentin

\section{INTRODUCTION}

Cervical cancer affects women worldwide and is the second most prevalent among gynecological cancers. Incidence rates vary across the planet and are higher in developing countries (Bray et al., 2018). Estimates range from 28.4 / 100,000 inhabitants in developing countries to 6.5 / 100,000 inhabitants in more developed regions (Bray et al., 2018). There is a wide variation in the mortality rate in the various regions, ranging from $2.2 / 100,000$ inhabitants, in developed regions such as Western Europe, Western Asia, Australia and New Zealand to 19.9 / 100,000, in countries such as Malaysia and Sub-Saharan Africa (Vaccarella et al., 2017; Bray et al., 2018).

The main risk factor for cervical cancer is persistent infection with human papillomavirus (HPV), mainly by genotypes 16 and 18 (Doobar, 2006). The expression of HPV oncoproteins leads to changes in tumor suppressor proteins such as p53 and retinoblastoma protein $(\mathrm{pRb})$, altering the cell cycle and modifying a series of signaling pathways that favor tumor growth (Doorbar, 2006; McCloskey et al., 2010; Monsonego et al., 2011; Husain et al., 2016). With progression of the disease, tumor cells rupture the basal membrane of the epithelium, leading to growth of the primary tumor and its subsequent spread to distant tissues resulting in invasion and metastasis (Husain et al., 2016). Invasion and metastasis processes, in turn, include a sequence of events, such as migration of tumor cells to adjacent or distant sites, intravasation of these cells into the bloodstream, survival of tumor cells in the circulatory system, displacement and extravasation by the vascular wall towards the parenchyma, and formation of colonies and micro-metastases in the parenchyma, which may progress to metastatic lesions (Banyard and Bielenberg, 2015; Chapman et al., 2015; Pattabiraman and Weinberg, 2016; Brabletz et al., 2018).

In the process of tumor propagation, reactivation of the epithelial-mesenchymal transition (EMT) program is observed, leading to alterations in the expression of genes that code for transcription factors (Shibue and Weinberg, 2017), such as those of the SNAIL, TWIST and $Z E B$ families (Brabletz et al., 2018). EMT involves increasing the invasion potential of tumor cells with decreased cell adhesion and increased ability for invasion and migration, as well as cellular morphological alterations, including tumor cell cytoskeletal rearrangements (Husain et al., 2016). In this reprogramming, there is a decrease in adhesion proteins, E-cadherins, and increased expression of Vimentin, $57 \mathrm{kDa}$ protein encoded by a single copy of a gene, called VIM, located on the chromosome 10p13 (Yamashita et al., 2013; Figiel et al., 2017).

Vimentin confers mechanical resistance to cells; however, although its structural role (Ridge et al., 2016) has long been considered as the main function of this protein, it is now 
known to play an important role in signal transduction pathways, and can interact with a vast network of proteins and therefore act on the positioning of organelles, migration, adhesion, and cell signaling (Dave and Bayless, 2014; Pérez-Sala et al., 2015). Vimentin is not normally expressed in epithelial cells, which make its unusual expression a biomarker of interest (Sitole and Mavri-Damelin, 2018). Studies have investigated the association of vimentin with some types of human cancers, such as prostate and breast cancers (Yamashita et al., 2013; Zhao et al., 2014; Du et al., 2018). Aberrant expression of vimentin and its association with metastatic potential in cervical cancer has been investigated by some authors (Gilles et al., 1996; Cheng et al., 2012; Yu et al., 2015; Husain et al. 2016); however, there have been few such studies. We investigated a possible association between vimentin expression and clinical-pathological aspects and prognosis of cervical cancer.

\section{MATERIAL AND METHODS}

\section{Selection of cases}

The study was approved by the Ethics Committee of Hospital Araújo Jorge, GoiâniaGoiás, Brazil. It was a retrospective study that included 111 cases of cervical carcinoma, 84 squamous carcinomas, 17 adenocarcinomas and 10 adeno-squamous carcinomas, distributed among the various clinical stages (Tis, I, II, III, IV). The histopathological diagnoses were performed in 2006 and the cases were selected from the database of the Pathology Department of Hospital Araújo Jorge. The socio-demographic and clinical-pathological data were obtained through an active search in the medical records. Cases included in the study had a confirmed histopathological diagnosis of cervical cancer, tumor-containing paraffin blocks, complete sociodemographic and clinical-pathological data, and tumor specimens obtained prior to radiotherapy.

\section{Immunohistochemistry (IHC)}

The immunohistochemical analysis of vimentin expression used the monoclonal antibody (anti-vimentin, clone V9, DAKO-AGILENT, dilution 1:2) and polymer-associated immunoperoxidase methods (Novolink-Novocastra Max Polymer Detection System commercial kit - Leica). Tumor slices were mounted on silanized slides, subsequently dewaxed in xylol at controlled temperature, and then rehydrated in a series of washes with ethanol $(100 \%, 80 \%$ and $50 \%$ ). They were then submitted to antigenic recovery by moist heat in an electric pressure cooker for seven min using $10 \mathrm{mM}$ Citrate / $\mathrm{pH}$ 6.0. After antigen retrieval, the slides were kept at room temperature for cooling for about $20 \mathrm{~min}$ and washed in phosphate buffered saline (PBS). Blocking of the endogenous peroxidase was done in hydrogen peroxide $10 \mathrm{~V}$ at $3 \%$ for 10 min, and thereafter, the slides were washed with PBS. After blocking, they were incubated at $4^{\circ} \mathrm{C}$ overnight with the primary antibody, washed with PBS and incubated for 30 min with the post-primary reagent, then washed again with PBS and incubated for an additional 30 min with the polymer containing dextran core. After a further wash with running water and PBS, the reaction was developed with 3-3'diaminobenzidine tetrahydrochlorate (DAB) for $10 \mathrm{~min}$, and lightly counter-stained with Harris hematoxylin. At that time, the slides were dehydrated in alcohol and xylol and mounted with coverslips using Entellan New (Merck Millipore). A slice of melanoma tissue was used as a positive control, which was included in each reaction performed. All reactions were processed under the same environmental conditions.

The expression of vimentin was evaluated by the presence of brown granules in the cytoplasm of the cells. The final marking score was calculated using a semi-quantitative method 
based on the intensity and percentage of stained cells as described by Yu et al. (2015). Three highly marked fields were selected and considered for evaluation. The score was based on the percentage of labeled cells and divided into four groups: (1) when markers were observed in less than $10 \%$ of tumor cells; (2) between 11 and $40 \%$; (3) between 41 and 75\%; (4) with markers $\geq 76 \%$. The final analysis considered only the percentage of marked cells, since all cases showed high intensity marking. Vimentine marking scores were dichotomized into hypoexpressed cases with marking indices $\leq 40 \%$, and hyperexpressed cases with marking $>40 \%$ (Figure 1).

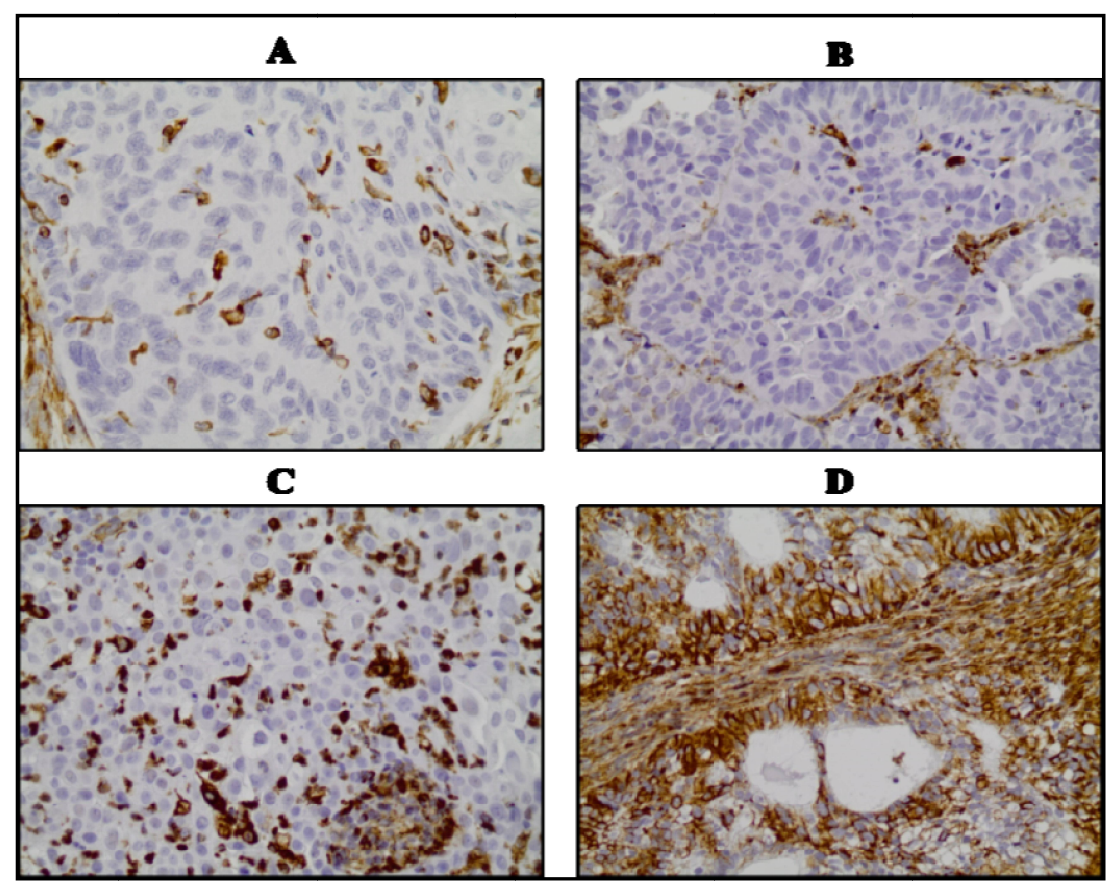

Figure 1. Vimentin expression score $\leq 40 \%$. (A) Squamous carcinoma. (B) Adenocarcinoma. Vimentin expression score $>40 \%$. (C) Squamous carcinoma. (D) Adenocarcinoma.

\section{Statistical analysis}

The absolute and relative frequencies of the categorical variables were calculated in relation to the non-categorical variables, calculations of central tendency, i.e., mean and median, and dispersion were calculated, including standard deviation and coefficient of variation. Subsequently, association tests were applied to perform comparative statistics. The test applied was Fisher's exact test. Finally, overall survival was calculated using the Kaplan Meier method. The data were stratified, and survivals were compared using the Log-Rank method, assuming pvalues less than 0.05 as significant.

\section{RESULTS}

The study group included 111 women with cervical cancer with histopathological confirmation. Clinical and sociodemographic data are described in Table 1. The age of the women in the group ranged from 26 to 88 years and the mean age was 51.2 years $( \pm 15.3)$. Less extensive tumors (Tis + T1) were the most common, accounting for $65 \%$ of the cases. 
Table 1. Sociodemographic and clinicopathological characteristics of patients with cervical cancer.

\begin{tabular}{|c|c|}
\hline \multirow{2}{*}{\multicolumn{2}{|c|}{$\begin{array}{l}\text { Variable } \\
\text { Age Group }\end{array}$}} \\
\hline & \\
\hline$\leq 40$ years & 34.2 \\
\hline$>40$ years & 65.8 \\
\hline \multicolumn{2}{|l|}{ Civil Status } \\
\hline Single & 22.5 \\
\hline Other & 77.5 \\
\hline \multicolumn{2}{|l|}{ Smoker } \\
\hline Yes & 38.7 \\
\hline No & 51.4 \\
\hline No information & 9.9 \\
\hline \multicolumn{2}{|l|}{ Menstrual Status } \\
\hline Menacme & 49.5 \\
\hline Menopause & 50.5 \\
\hline \multicolumn{2}{|l|}{ Surgical Treatment } \\
\hline Yes & 61.3 \\
\hline No & 38.7 \\
\hline \multicolumn{2}{|c|}{ Radiotherapeutic Treatment } \\
\hline External Radiotherapy & 13.5 \\
\hline Brachytherapy & 0.9 \\
\hline Both & 34.2 \\
\hline None & 51.4 \\
\hline \multicolumn{2}{|l|}{ Chemotherapy } \\
\hline Yes & 9.9 \\
\hline No & 90.1 \\
\hline \multicolumn{2}{|l|}{ Clinical TNM Stage } \\
\hline Tis & 3.6 \\
\hline I & 56.8 \\
\hline II & 14.4 \\
\hline III & 9.9 \\
\hline IV & 12.6 \\
\hline NA & 2.7 \\
\hline \multicolumn{2}{|l|}{ Size of Tumor (T) } \\
\hline Tis & 6.3 \\
\hline T1 & 58.6 \\
\hline $\mathrm{T} 2$ & 18.9 \\
\hline T3 & 9.0 \\
\hline $\mathrm{T} 4$ & 7.2 \\
\hline \multicolumn{2}{|c|}{ Lymph node metastasis $(\mathbf{N})$} \\
\hline No & 87.4 \\
\hline N1 & 3.6 \\
\hline Nx & 9.0 \\
\hline \multicolumn{2}{|l|}{ Distant Metastasis (M) } \\
\hline M0 & 89.2 \\
\hline M1 & 9.9 \\
\hline $\mathrm{Mx}$ & 0.9 \\
\hline \multicolumn{2}{|l|}{ Histological Type } \\
\hline Squamous Cell & 75.7 \\
\hline Adenocarcinoma & 15.3 \\
\hline Adenosquamous & 9.0 \\
\hline \multicolumn{2}{|l|}{ Death Reported } \\
\hline Alive & 65.8 \\
\hline Death Reported & 34.2 \\
\hline
\end{tabular}

Vimentin expression was observed in $100 \%$ of the tumors evaluated and its quantification resulted in two groups of tumors: tumors with expression $\leq 40 \%$ (hypoexpression) and tumors with expression $>40 \%$ (hyperexpression) (Table 2). Based on this classification, it 
was observed that $5 \%$ of the cervical carcinomas presented hypoexpression of vimentin. Vimentin expression was investigated in relation to the clinical-pathological variables of the tumors, including size, lymph node metastasis, distant metastasis, clinical staging, histological type and recorded death; however, no significant difference was observed among the variables. Expression of vimentin detected by immunohistochemistry was observed in the histological sections, which accompany the respective hematoxylin-eosin stained sections (Figures 2 and 3).

Table 2. Vimentin expression in relation to the clinical-pathological characteristics of carcinomas of the cervix.

\begin{tabular}{|c|c|c|c|}
\hline & $\begin{array}{l}\text { Vimentin Expression } \\
\leq 40 \%(n=83)\end{array}$ & $>40 \%(n=28)$ & p-value \\
\hline Variable & $f(\%)$ & $f(\%)$ & \\
\hline \multicolumn{4}{|l|}{ Size of Tumor (T) } \\
\hline $\mathrm{T}$ is $+\mathrm{T} 1+\mathrm{T} 2$ & 84.3 & 82.1 & \\
\hline $\mathrm{T} 3+\mathrm{T} 4$ & 15.7 & 17.9 & 0.772 \\
\hline \multicolumn{4}{|l|}{ Lymph node metastasis $(\mathrm{N}) *$} \\
\hline No & 96.1 & 96.0 & \\
\hline N1 & 3.9 & 4.0 & 0.954 \\
\hline \multicolumn{4}{|l|}{ Distant Metastasis $(\mathbf{M})^{* *}$} \\
\hline M0 & 90.2 & 89.3 & \\
\hline M1 & 9.8 & 10.7 & 0.994 \\
\hline \multicolumn{4}{|l|}{ TNM Clinical Stage ${ }^{* * *}$} \\
\hline Tis + I + II & 77.5 & 75.0 & \\
\hline $\mathrm{III}+\mathrm{IV}$ & 22.5 & 25.0 & 0.798 \\
\hline \multicolumn{4}{|l|}{ Histological Type } \\
\hline Squamous Cell & 77.1 & 71.4 & \\
\hline Adenocarcinoma + Adenosquamous & 22.9 & 28.6 & 0.613 \\
\hline \multicolumn{4}{|l|}{ Status or Death Registered } \\
\hline Alive & 61.4 & 78.6 & \\
\hline Death Registered & 38.6 & 21.4 & 0.113 \\
\hline
\end{tabular}

Excluded: $(*) 10 \mathrm{Nx} ;(* *) 1 \mathrm{Mx} ;(* * *) 3$ Not evaluated. Test Used: Fisher's Exact Test

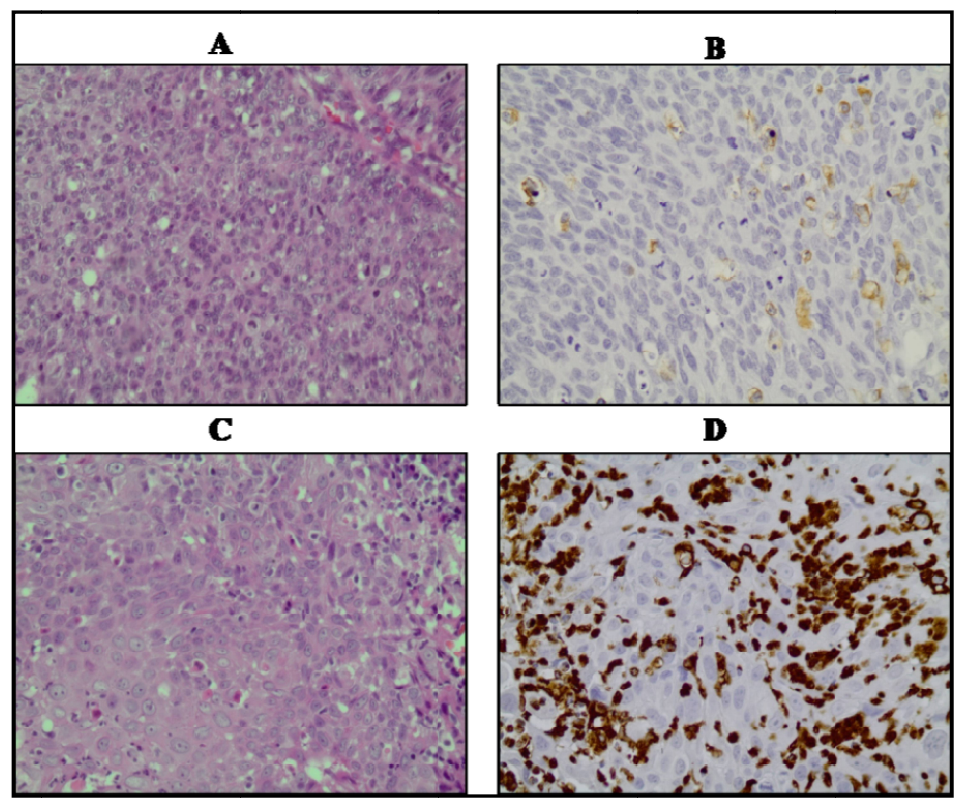

Figure 2. A and C. Adenocarcinoma stained with hematoxylin-eosin (HE). B Immunoexpression of vimentin in invasive adenocarcinoma $\leq 40 \%$ and. D immunoexpression of vimentin $>40 \%$. 


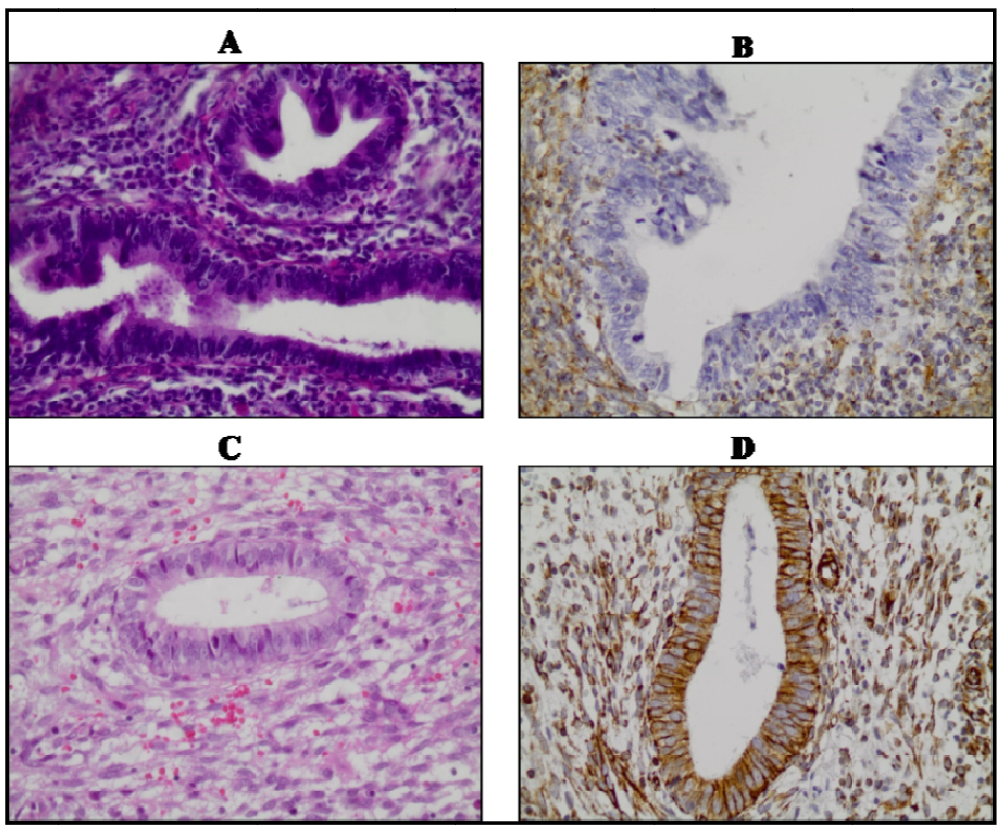

Figure 3. $\mathrm{A}$ and $\mathrm{C}$ Adenocarcinoma stained with hematoxylin-eosin). $\mathrm{C}$ Immunoexpression of vimentin in invasive adenocarcinoma $\leq 40 \%$ and. D immunoexpression of vimentin $>40 \%$.

\section{Survival analysis}

Overall survival at 60 months for the study group was 66\%, (Figure 4). Survival was greater for patients aged $\leq 50$ years $(84 \%)(\mathrm{P}<0.0001)$ (Figure 4$)$. Regarding histological type, there were no differences in survival between the groups evaluated $(\mathrm{P}=0.585)$ (Figure 5). Regarding the extent of the lesion, survival was higher $(76 \%)$ for patients presenting with smaller tumors (Tis, T1, T2) $(\mathrm{P}<0.0001)$ (Figure 6).

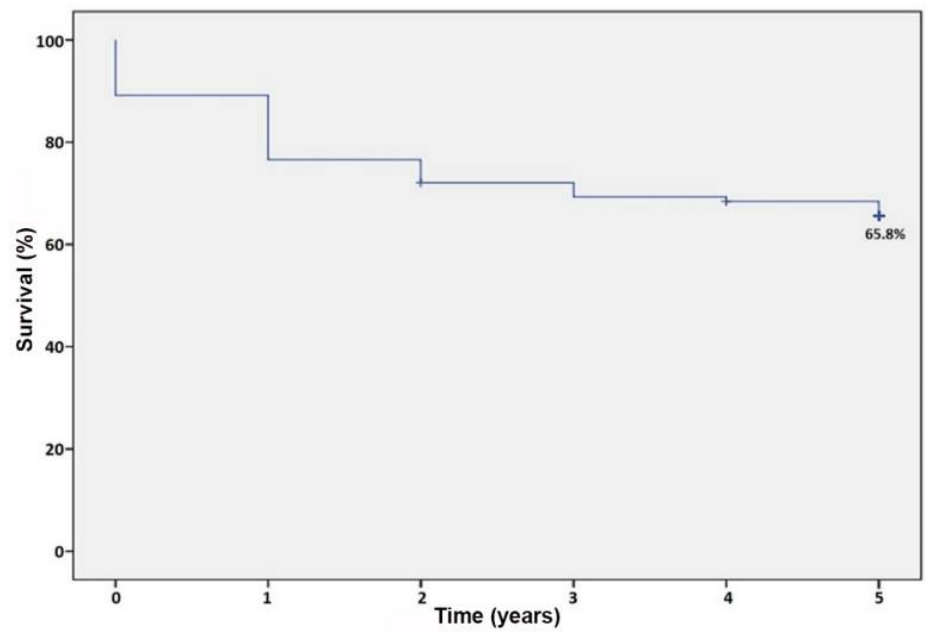

Figure 4. Five- year survival for the group studied at 60 months. 


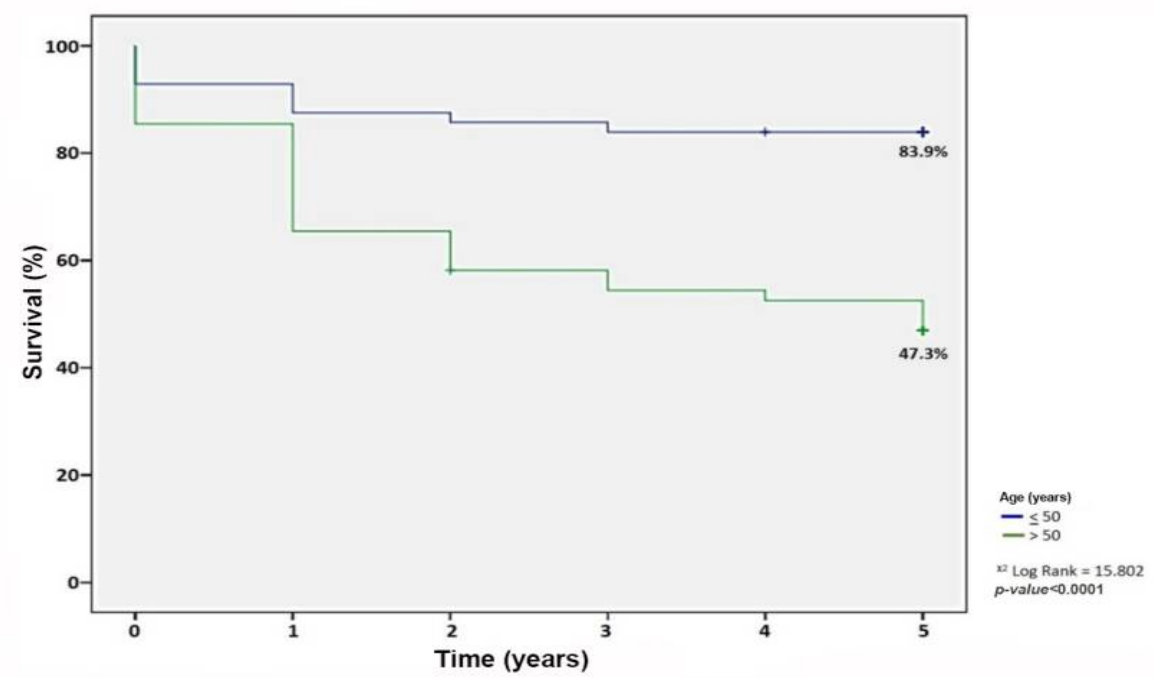

Figure 5. Five-year survival for patients with cervical cancer by age.

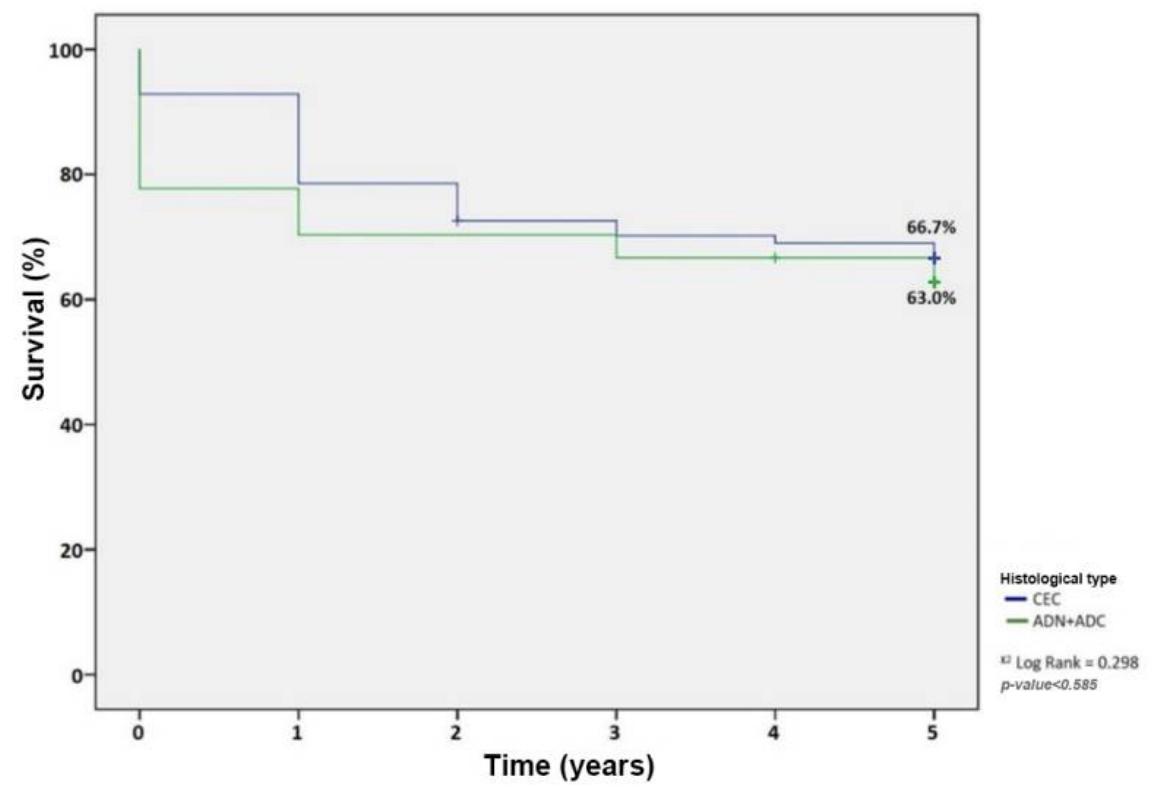

Figure 6. Five-year survival for patients with uterine cervix cancer by histological type.

Regarding the presence of lymph node metastasis, survival was higher (75.3\%) for patients with non-metastatic tumors compared to lymph node metastases $(\mathrm{P}<0.0001)$ (Figure 7). In the case of the presence of distant metastasis, survival was greater (73.7\%) for patients with non-metastatic tumors and all those with distant metastasis died during the period evaluated $(\mathrm{P}<0.0001)$ (Figure 8). In relation to TNM staging, survival was greater (80.7\%) for those with less advanced stages (Tis, TI and TII), compared to those with more advanced staging (TIII and TIV), which was $12 \%$ (P < 0.0001) (Figure 9). 


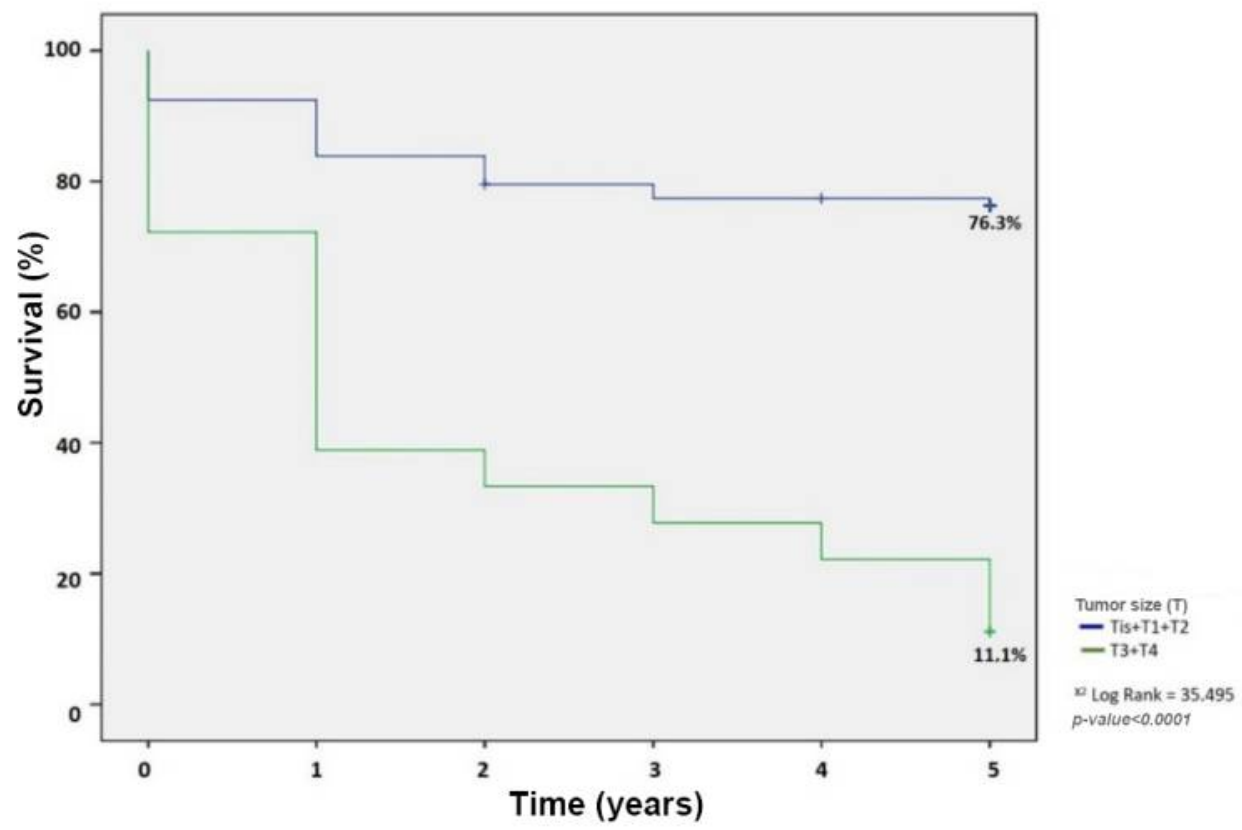

Figure 7. Five- year survival in five for patients with cervical cancer by disease extension.

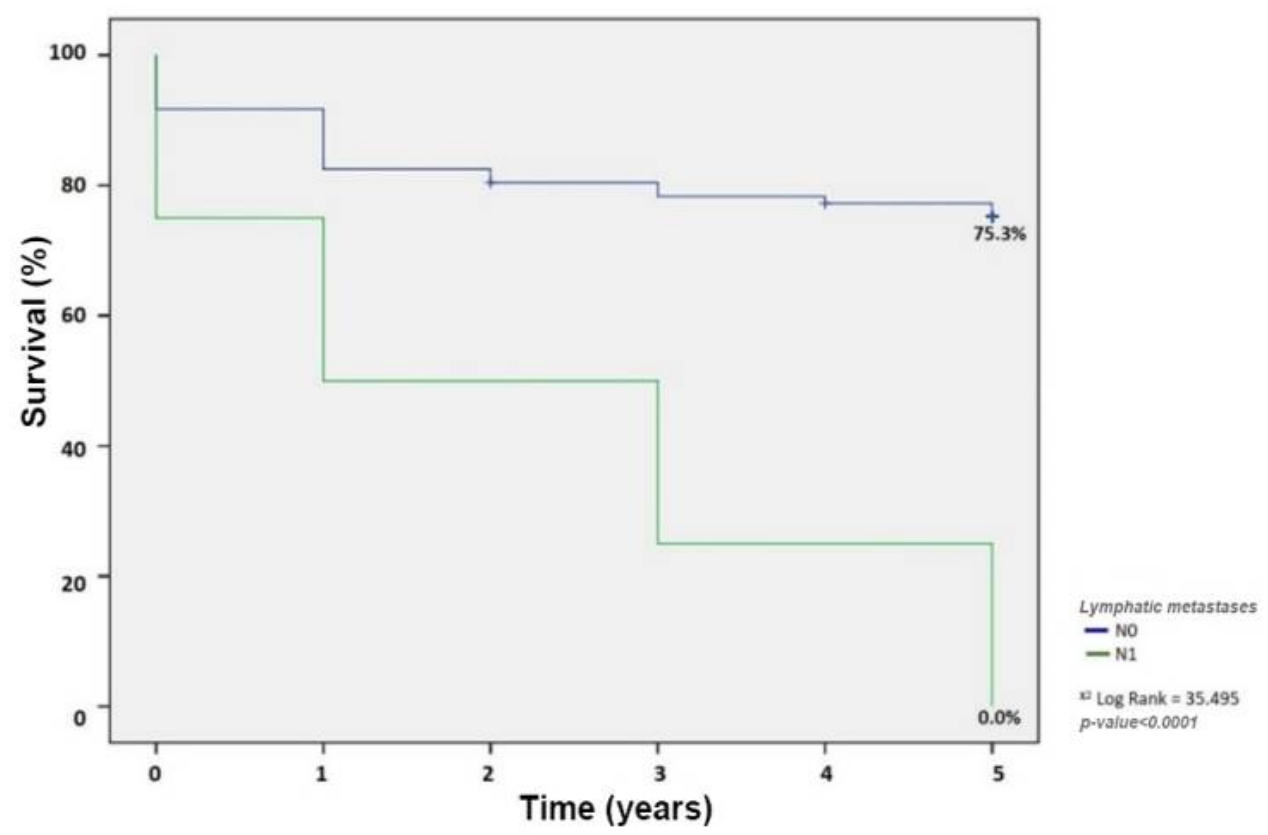

Figure 8. Five-year survival for patients with cervical cancer by the presence of lymph node metastasis. 


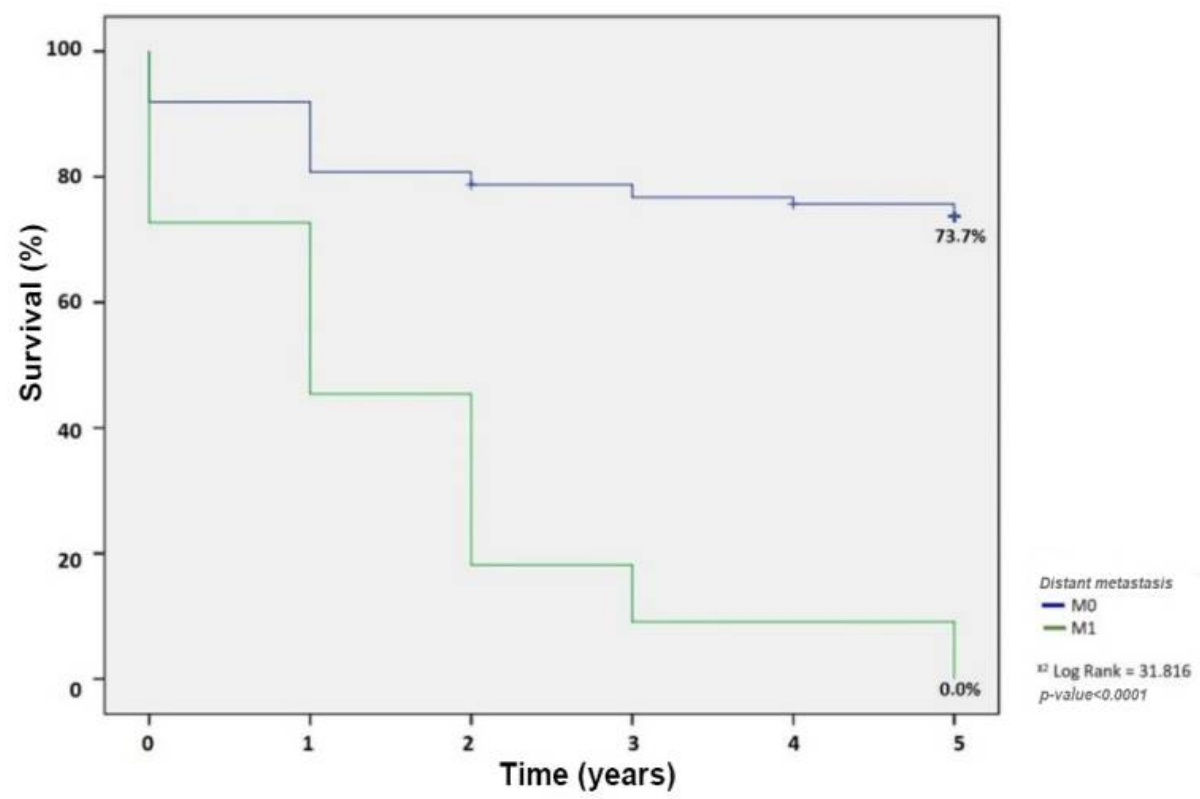

Figure 9. Five-year survival for patients with cervical cancer by the presence of distant metastasis.

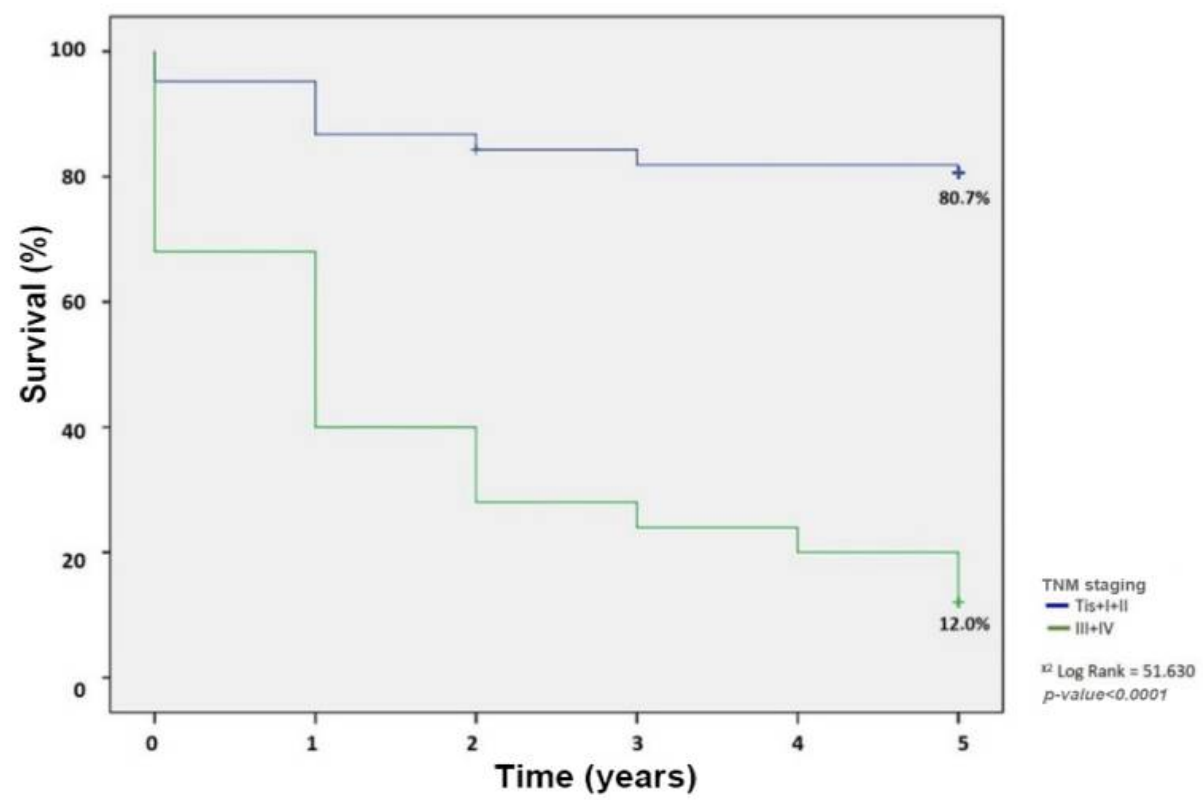

Figure 10. Five-year survival for patients with cervical cancer by TNM staging.

On the expression of vimentin, survival was $61.4 \%$ for patients with hypoexpression and $78.6 \%$ for those with hyperexpression. There was no difference in survival between the groups evaluated $(\mathrm{P}=0.105)$ (Figure 11). 


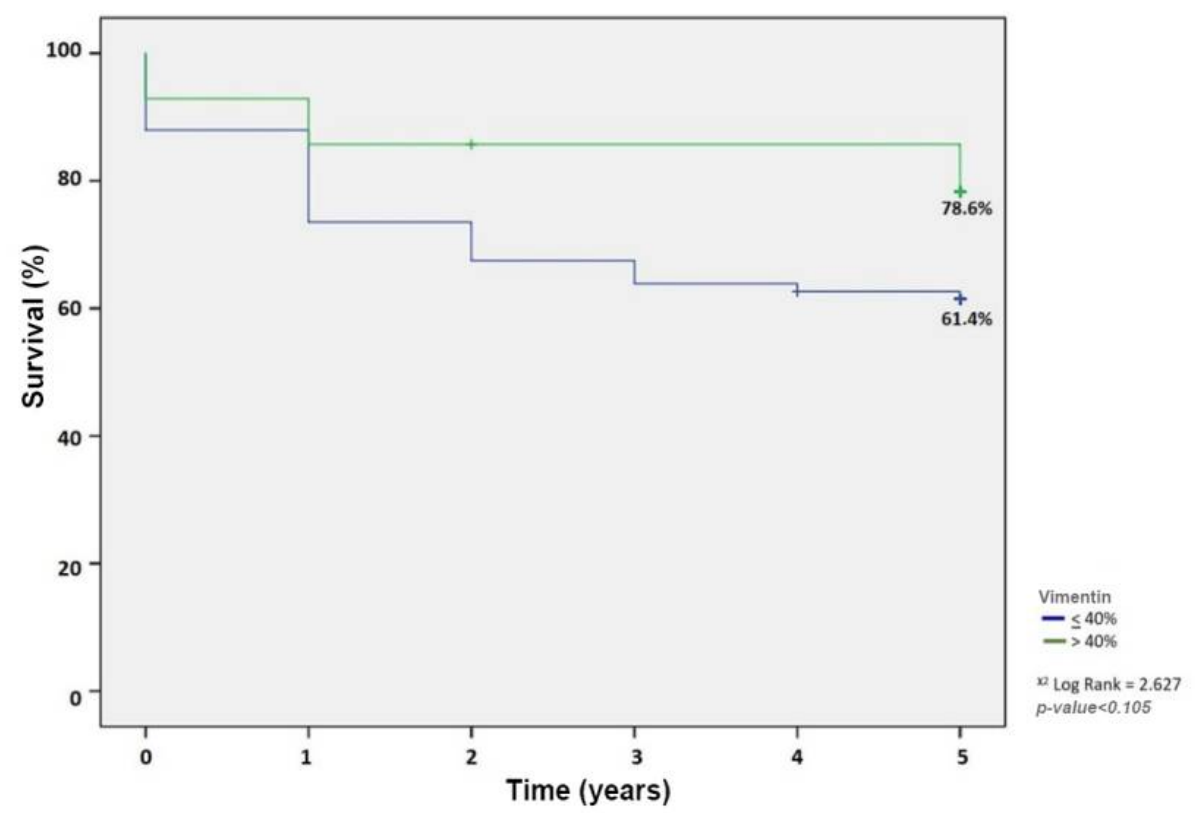

Figure 11. Five-year survival for patients with cervical cancer by vimentin expression.

\section{DISCUSSION}

The unusual expression of vimentina during reactivation of the EMT program in the invasion and metastasis process has already been investigated in several human tumors (Zhao et al., 2014; Holthoff et al., 2016; Figiel et al., 2017; Du et al., 2018). Regarding cervical cancer, there are still few studies evaluating the association of vimentin expression with prognostic aspects of tumors, especially with regard to survival and relapse-free interval evaluations (Gilles et al., 1996; Cheng et al., 2012; Yu et al., 2015; Husain et al., 2016; Lin et al., 2017).

In the 111 cases of cervical cancer investigated in this study using immunohistochemistry, Vimentin expression was observed in $100 \%$ of the tumor specimens, confirming the unusual expression of this protein in both squamous carcinomas and adenocarcinomas as well as adeno-squamous carcinomas of the uterine cervix. This finding is not usually found in the cervical epithelial cells, but only in the mesenchymal ones, where the cytoskeleton network is formed, composed of robust filaments containing vimentin, which extend from the nuclear periphery to the cell membrane, giving a greater mechanical resistance to the cells (Satelli and Li, 2011; Pattabiraman and Weinberg, 2016; Ridge et al., 2016). Vimentin acts to control the activity of proteins involved in cell migration, in some cases protecting the degradation mediated by the ubiquitin-proteasome system. Therefore, the hyperexpression of vimentin during the EMT event seems to promote greater stabilization and a greater cellular migration, increasing the invasive capacity of the cells, this characteristic leads to a worse prognosis for tumors (Phua et al., 2009; Satelli and Li, 2011).

The initial hypothesis of our study was that the hyperexpression of vimentin would be associated with the factors of worse prognosis in cervical cancer and, consequently, the shorter survival of the patients. After the investigation of the possible associations between the expression of vimentin and the classic prognostic factors for cervical cancer, as well as the 
survival of the patients, this hypothesis was not confirmed. Therefore, our results differ from those reported in previous studies such as (Cheng et al., 2012; Yu et al., 2015; Husain et al., 2016; Lin et al., 2017).

These differences may result from the different methodologies used. In some studies (Gilles et al., 1994; Gilles et al., 1994; Holthoff et al., 2016), the association between vimentin expression and the invasive properties of cervical cancer was initially studied in models of cervical cell lines transformed by HPV (Gilles et al., 1994; Gilles et al., 1994; Holthoff et al., 2016). The association between vimentin expression and tumor invasion was reported, comparing to the expression of vimentin by immunohistochemistry in cases of cervical carcinomas in situ and invasive cervical carcinomas (Holthoff et al., 2016). This same study evaluated metastatic lesions of cervical carcinomas and demonstrated the expression of vimentin in metastases, but to a lesser extent (Holthoff et al., 2016). Another author (Cheng et al., 2012) evaluated 135 patients with squamous cell cervical cancer in stages I and II and demonstrated that $65 \%$ of the cases were positive for vimentin expression. Vimentin expression was inversely associated with histological differentiation of tumors, presence of lymph node metastasis and recurrence of cancer. In univariate and multivariate analysis, hyperexpression of vimentin (above $80 \%$ of labeled cells) was considered a worse prognostic factor, since it was significantly associated with shorter survival of patients with squamous cell carcinoma of the cervix (Cheng et al., 2012). However, the study did not evaluate more advanced tumors in stages III and IV (Cheng et al., 2012).

Proteins related to the epithelial-mesenchymal transition, including vimentin, were investigated in in-situ carcinomas (81 cases), squamous microinvasive carcinomas (17 cases) and squamous invasive carcinomas (21 cases) to demonstrate their key roles in the progression of tumors. The study was performed on paraffin blocks included in a microarray and the expression of vimentin was higher in microinvasive and invading carcinomas when compared to carcinomas in situ. However, the clinical-pathological and prognostic aspects of the tumors were not evaluated in the study (Myong, 2012).

A case-control study (Husain et al., 2016) compared the expression of vimentin with the clinical-pathological features of tumors, including tumor grade and age of patients with cervical cancer. The cytoplasmic expression of vimentin was observed in $40 \%$ of the cases and was negative in benign non-tumoral inflammatory lesions of the cervix. Vimentin expression was significantly associated with tumor grade and did not vary according to the different age groups. The results demonstrated a possible role of vimentin in the development and progression of cervical cancer however, other clinical-pathological and prognostic aspects were not investigated in the study (Husain et al., 2016). One study (Satelli and $\mathrm{Li}, 2011$ ) investigated the prognostic value of vimentin expression in cervical cancer, associating clinical-pathological factors with other markers, such as TP53. The score used by the authors was $10 \%$, considering hypoexpression below $10 \%$ and hyperexpression above $10 \%$ (Satelli and $\mathrm{Li}, 2011$ ).

Several differences can be pointed out between this study and others. First, with respect to the primary anti-vimentin antibodies that were used. Secondly, the procedures of microscopic evaluation of the expression of vimentin and the cut-off points used to define hypo and hyperexpression of this biomarker. The immunohistochemistry method is relatively simple but exhibits particularities that may influence results, including tumor specimen fixing time, antigen retrieval method, and the choice of antibodies and reagents used. The analysis of vimentin expression by immunohistochemistry is widely used, however, it presents many challenges and difficulties, including reproducibility (Kosti et al., 2016), lack of standardization with regard to the type of antibody used, and the method of microscopic evaluation of the tissue marking. All this variability may have influenced the diversity of results found in the available studies. In 
addition, our study included the different histological types of cervical cancer and different clinical stages, which may have made it difficult to detect possible associations between the expression of vimentin and the prognosis of these tumors.

An important aspect to be considered is the lack of standardization of semiquantitative evaluation methods and the different cutoff points used to define hypoexpression or hyperexpression of vimentin in the studies (Gilles et al., 1996; Cheng et al., 2012; Yu et al., 2015; Husain et al., 2016). In this study, the criteria of Yu et al. was used, which consider the index and intensity of the marker, however, it is necessary to point out that all the samples had high marker intensity, and for the statistical analysis of the results, marker indices were dichotomized.

The five-year overall survival of the studied group was $65.8 \%$, similar to another study evaluated in another Brazilian region in the same period (Mascarello et al., 2013; Alves et al., 2017). Although not influenced by the expression of vimentin, survival was affected by classical prognostic factors, such as tumor size ( $\mathrm{p}<0.0001)$, lymph node metastases $(\mathrm{p}<0.0001)$, and distant metastases $(\mathrm{p}<0,0001)$, which validates the casuistry and the data collected. Although the results did not show significant associations between the expression of vimentin and the prognostic factors of cervical carcinomas, aberrant expression of vimentin was observed in all the cases studied, including squamous carcinomas, adenocarcinomas and adenosquamous carcinomas, characterizing EMT in these tumors, as well as the potential for invasion and metastasis of this type of cancer (Satelli and Li, 2011; Pattabiraman and Weinberg, 2016).

In conclusion, the expression of vimentin was observed in all cases of cervical cancer evaluated and expression level was not associated with the clinical-pathological the characteristics of the tumors analyzed in this study.

\section{ACKNOWLEDGMENTS}

The authors thank Hospital Araujo Jorge and the Pathology Department team. Brian Ream edited the English language. The authors thank FAPEG, Fundação de Amparo à Pesquisa do Estado de Goias, for financial support.

\section{CONFLICTS OF INTEREST}

The authors declare no conflict of interest.

\section{REFERENCES}

Banyard J and Bielenberg DR (2015). The role of EMT and MET in cancer dissemination. Connect Tissue Res. 56: 403413.

Brabletz T, Kalluri R, Nieto MA and Weiberg RA (2018). EMT in cancer. Nat. Rev. Cancer. 18: 128-134.

Bray F, Ferlay J, Soerjomataram I, Siegel RL, et al. (2018). Global cancer statistics 2018: GLOBOCAN estimates of incidence and mortality worldwide for 36 cancers in 185 countries. CA. Cancer J. Clin. 68: 394-424.

Cheng Y, Zhou Y, Jiang W, Yang X, et al. (2012). Significance of E-cadherin, $\beta$-catenin, and vimentin expression as postoperative prognosis indicators in cervical squamous cell carcinoma. Human. Pathol. 43: 1213-1220.

Dave JM and Bayless KJ (2014). Vimentin as an integral regulator of cell adhesion and endothelial sprouting. Microcirculation. 21: 333-344.

Doorbar J (2006). Molecular biology of human papillomavirus infection and cervical cancer. Clin. Sci. (Lond). 110: 525541.

Du L, Li J, Lei L, He H, et al. (2018). High Vimentin expression predicts a poor prognosis and progression in colorectal cancer: a study with meta-analysis and TCGA database. Biomed. Res. Int. 2018: 6387810.

Figiel S, Vasseur C, Bruyere F, Rozet F, et al. (2017). Clinical significance of epithelial-mesenchymal transition markers in prostate cancer. Hum. Pathol. 61: 26-32. 
Gilles C, Piette J, Peter W, Fusenig NE, et al. (1994). Differentiation ability and oncogenic potential of HPV-33 and HPV-33+ras-transfected keratinocytes. Int. J. Cancer. 58: 847-54.

Gilles C, Polette M, Piette J, Birembaut P, et al. (1996). Epithelial-to-mesenchymal transition in HPV-33-transfected cervical keratinocytes is associated with increased invasiveness and expression of gelatinase. A. Int. J. Cancer. 59: 661-6.

Gilles C, Polette M, Piette J, Delvigne AC, et al. (1996). Vimentin expression in cervical carcinomas: Association with invasive and migratory potential. J. Pathol. 180: 175-80.

Husain NE, Babiker AY, Albutti AS, Alsahli MA, et al. (2016). Clinicopathological significance of Vimentin and Cytokeratin protein in the genesis of squamous cell carcinoma of cervix. Obstet. Gynecol. Int. 2016: 8790120.

Kosti I, Jain N, Aran D, Butte AJ, et al. (2016). Cross-tissue analysis of gene and protein expression in normal and cancer tissues. Sci. Rep. 6: 24799.

Lambert AW, Pattabiraman DR and Weinberg RA (2017). Emerging Biological Principles of Metastasis. Cell. 168: 670691.

Lin J, Lu J, Wang C, Xue X (2017). The prognostic values of the expression of Vimentin, TP53, and Podoplanin in patients with cervical cancer. Cancer Cell Int. 17: 80 .

Mascarello KC, Zandonade E and Amorim MH (2013). Survival analysis of women with cervical cancer treated at a referral hospital for oncology in Espírito Santo State, Brazil, 2000-2005. Cad. Saude Publica. 29: 823-31.

McCloskey R, Menges C, Friedman A, Patel D, et al. (2010). Human Papillomavirus type 16 E6/E7 upregulation of nucleophosmin is important for proliferation and inhibition of differentiation. J. Virol. 84: 5131-5139.

Monsonego J., Hudgens MG, Zerat L, Zerat JC, et al. (2011). Evaluation of oncogenic human papillomavirus RNA and DNA tests with liquid-based cytology in primary cervical cancer screening: the FASE study. Int. J. Cancer. 129: 691-701.

Myong NH (2012). Loss of E-cadherin and acquisition of vimentin in epithelial-mesenchymal transition are noble indicators of uterine cervix cancer progression. Korean J. Pathol. 46: 341-8.

Nieto MA, Huang RY, Jackson RA and Thiery JP (2016). EMT:2016. Cell. 166: 21-45.

Pattabiraman DR and Weinberg RA (2016). Targeting the epithelial-to-mesenchymal transition: the case for differentiation-based therapy. Cold Spring Harb. Symp. Quant. Biol. 81: 11-19.

Phua DC, Humbert PO and Hunziker W (2009). Vimentin regulates scribble activity by protecting it from proteasomal degradation. Mol. Biol. Cell. 20: 2841-55.

Ridge KM, Shumaker D, Robert A, HooKway C, et al. (2016). Methods for determining the cellular functions of Vimentin intermediate filaments. Methods Enzymol. 568: 389-426.

Satelli A and Li S (2011). Vimentin in cancer and its potential as a molecular target for cancer therapy. Cell Mol. Life Sci. 68: 3033-46.

Sitole BN and Mavri-Damelin D (2018). Peroxidasin is regulated by the epithelial-mesenchymal transition master transcription factor Snail. Gene. 646: 195-202.

Shibue T and Weinberg RA (2017). EMT, CSCs, and drug resistance: the mechanistic link and clinical implications. Nat. Rev. Clin. Oncol. 14: 611-629.

Thiery JP, Acloque H, Huang RY and Nieto MA (2009). Epithelial-mesenchymal transitions in development and disease. Cell. 139: 871-90.

Vaccarella S, Laversanne M, Ferlay J and Bray F (2017). Cervical cancer in Africa, Latin America and the Caribbean and Asia: regional inequalities and changing trends. Int. J. Cancer. 141: 1997-2001.

Yamashita N. Tokunaga E, Kitao H, Hisamatsu Y, et al. (2013). Vimentin as a poor prognostic factor for triple-negative breast cancer. J. Cancer Res. Clin. Oncol. 139: 739-746.

Yu JQ, Zhou Q, Zheng YF and Bao Y (2015). Expression of vimentin and Ki-67 proteins in cervical squamous cell carcinoma and their relationships with clinicopathological features. Asian Pac. J. Cancer Prev. 16: 4271-4275.

Zhao J, Dong D, Sun L, Zhang G, et al. (2014). Prognostic significance of the epithelial-to-mesenchymal transition markers e-cadherin, vimentin and twist in bladder cancer. Int. Braz. J. Urol. 40: 179-189. 